\title{
P215: Tracking infections in outpatient hemodialysis unit in Saudi Arabia
}

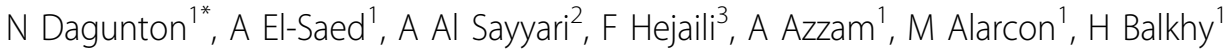 \\ From 2nd International Conference on Prevention and Infection Control (ICPIC 2013) \\ Geneva, Switzerland. 25-28 June 2013
}

\section{Introduction}

Bacteremias and localized infections of the vascular access site are associated with high morbidity and mortality among end-satge renal (ESRD) patients.

\section{Objectives}

At King Abdulaziz Medical City (KAMC) in Riyadh (SA), surveillance among ESRD patients was conducted to detect trends in dialysis related infections and evaluate effectiveness of prevention measures.

\section{Methods}

Following the methodology set by the National Healthcare Safety Network (NHSN), a 32-month prospective surveillance (monthly average of 218 patients-months) was conducted from 2008 to 2012 at the Outpatient Hemodialysis Unit at KAMC. Patients were monitored for any of the three dialysis events; outpatient start of an intravenous (IV) antimicrobial, evidence of local access site infection (LASI) and access-associated bacteremia.

Infection control measures were instituted throughout the whole period of study but stringent ones were strictly emphasized starting early 2012. Interventions implemented included the use of Chlorhexidine impregnated transparent dressings, strict monitoring of aseptic dressing technique, provision of an Arabic educational brochure for patient education, provision of an alcohol hand gel in each patient trolleys and judicious use of antibiotics.

\section{Results}

The combined rates of all three types of events significantly declined from 19.2 to 7.6 per 100 patients-months which constituted about $60 \%$ reduction. Comparing the rates between patients with AV fistulas and patients with permanent catheter, there was an $85 \%$ reduction in rates among patients with AV fistula (from 2.6 to .4) and 54\% decline in patients with permanent catheter (from 15.6 to 7.2). The rate of access-associated bacteremia among patients with AV fistula dropped from .5 to zero while the rate of patients with permanent catheter was reduced from 3.8 to 1.9 .

\section{Conclusion}

Tracking dialysis events and following basic infection control measures are indeed vital in the prevention of infections among ESRD patients. The surveillance has shown a significant decline in both AV fistula and permanent catheter but patients with AV fistula better responded to preventive measures.

\section{Disclosure of interest}

None declared.

\section{Author details}

${ }^{1}$ Infection Prevention \& Control, King Abdulaziz Medical City, Riyadh 11426, Saudi Arabia. ${ }^{2}$ Department of Nephrology, King Abdulaziz Medical City, Riyadh 11426, Saudi Arabia. ${ }^{3}$ Hemodialysis Program, King Abdulaziz Medical City, Riyadh 11426, Saudi Arabia.

Published: 20 June 2013

doi:10.1186/2047-2994-2-S1-P215

Cite this article as: Dagunton et al:: P215: Tracking infections in outpatient hemodialysis unit in Saudi Arabia. Antimicrobial Resistance and Infection Control 2013 2(Suppl 1):P215.

'Infection Prevention \& Control, King Abdulaziz Medical City, Riyadh 11426,

Saudi Arabia

Full list of author information is available at the end of the article

(c) 2013 Dagunton et al; licensee BioMed Central Ltd. This is an Open Access article distributed under the terms of the Creative Commons Attribution License (http://creativecommons.org/licenses/by/2.0), which permits unrestricted use, distribution, and reproduction in any medium, provided the original work is properly cited. 